\title{
Analysis of VoIP over Wired \& Wireless Network with Implementation of QoS CBWFQ \& 802.11e
}

\author{
Zahid Ali \\ Department of Telecommunication Engineering, Mehran University of Engineering and Technology \\ Jamshoro, Sindh, Pakistan \\ E-mail: zahid.ali@admin.muet.edu.pk \\ Falak Naz, Javed, Muhammad Qurban, Muhammad Yasir, Shehroz Jehangir \\ Department of Telecommunication Engineering, Mehran University of Engineering and Technology \\ Jamshoro, Sindh, Pakistan \\ E-mail: f16t192@students.muet.edu.pk, javedazeemikk@gmail.com, qurban18csn03@gmail.com, \\ yasirshaikh671@gmail.com and shehrozjehangir@gmail.com
}

Received: 28 November 2019; Accepted: 23 January 2020; Published: 08 February 2020

\begin{abstract}
In this paper, we analyzed VoIP data rates to minimize the bandwidth efficiently as per user demand and reduced the budget cost before implementing VoIP service at any wired and wireless network. To accomplish these results different clock rates were used to assign bandwidth administratively, CODEC schemes (G.711 and G.729) to minimized data rates and QoS (Quality of Service) such as CBWFQ and 802.11e to sustain the voice quality in congestion over the wired and wireless networks. PRTG Grapher and LAN Traffic Generator software were used to monitor a bandwidth and create congestion artificially between the link of communicating two setup LANs wired and wireless.
\end{abstract}

Index Terms-ATA (Analog Telephone Adapter), QoS (Quality of Service), PRTG (Paessler Router Traffic Grapher), WFQ (Weighted Fair Queuing) and AP (Access Point).

\section{INTRODUCTION}

Voice over Internet Protocol (VoIP) is very popular in recent days and has become the first choice for national to multinational companies for the integration of voice and data in order to reduce the cost and to use the IT resources is used in a much more efficient way. Another popular technology that is ruling the world since the year 2000 is 802.11 wireless networks. Organizations want to implement the VoIP on the wireless network because of its different nature and requirement than wired [1]. One of the major differences between wired and wireless is bandwidth availability. When we implement the VoIP, we must use the available bandwidth in an efficient way so that VoIP application will prove to be the best choice in using less bandwidth and sustaining the good voice quality [2]. Voices over the Internet Protocol is a modern high-tech technology that allows users to make phone calls via the Internet using a packet-switching network rather than a Public switched telephone network (PSTN). With the establishment of VoIP, today's digital communication has significantly decreased costs while maintaining a high quality of service (QoS) such as CBWFQ in wired and newly introduced 802.11e IEEE wireless standard. VoIP has also demonstrated its popularity in Ethernet LANs. Ethernet is a data networking platform that is used for businesses and other organizations, as it provides its users with a high quality of services and greatly reduces costs in comparison with the conventional PSTN. Wireless network (IEEE 802.11) also provides digital communication for customers from distant places, making it suitable for customers who work in offices, companies, institutions, hospitals and so on [3]

\section{LITERATURE REVIEW}

To establish Local Area Network Communication different organizations and companies require Ethernet because of its data networking platform which is considered not only the ideal platform but it also presents cost-efficient and great quality of service as compare to PSTN. Other than that for distant locations, distance digital communication is provided by wireless Ethernet networks for the connection of user and network [4].

\section{A. VoIP Over Wired Network}

Calls through Voice over Internet Protocol be it voice call or video call, are quite simple to be placed. Voice over Internet Protocol brings ease to ISP's and clients [5] with that it also lessens the burden by keeping it aside.

\section{a. VoIP Call using ATA}

To be carried on the Internet in the form of data 
packets, analog signals need to be converted in the digital signal which is done through Analog Telephone Adapters. Adapters are connected to the dial-up internet account through built-in modem and PSTN which through internet routes the call to the caller [3].

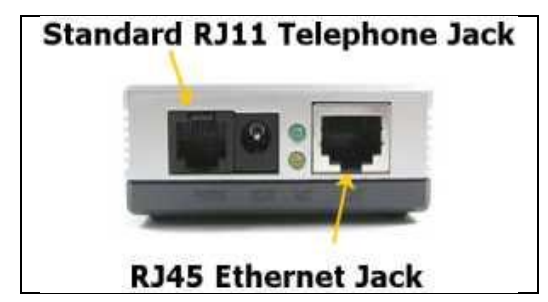

Fig.1. ATA

As shown in above Fig.1 Analog Telephone Adapters possess RJ-11 jack to be connected on the telephone and second is RJ-45 to be connected on Ethernet. This sort of ATA mostly operates with certain kinds of software.

\section{b. VoIP Call using IP Phones}

The phone system user of Voice over Internet Protocol merely connects his IP to the closest Local Area Network port. Then the IP-phone as given in Fig.2 automatically enters itself with the VoIP phone system. Numbers are kept the same of IP phones all the time and work precisely similar, regardless of where you connect to your desk or office. A SIP phone based on hardware is similar to an ordinary telephone. These devices have an embedded mini-hub, so you do not need an extra network point for the telephone to share a network link with the computer [6].

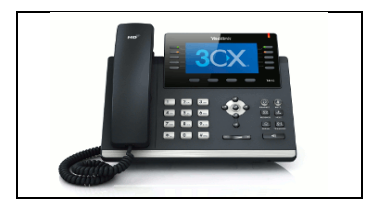

Fig.2. IP Phone

\section{B. VoIP Over Wireless Network}

Mobility is the main reason for the expansion of VoIP technology. The network of wireless Internet Protocol offers its users a wide variety of mobility as well as the specific features of the wireless network. It is a more difficult task for the traffic to be carried in real-time into packets with wireless networks, given that VoIP is a kind of application that works in real-time and is sensitive to the losses of packets due to external interference, coverage limitation and other devices using the similar range of frequency in the networks which are wireless [7]. For deploying VoIP over wireless networks efficiently voice transmission will need some specific requirements. Due to its delay sensitivity and the competition in the transmission of data through similar media as wireless, it results in the degradation of voice quality. It is therefore essential to enforce QoS properly [8] in wireless networks so that voice is given priority over data packets.

\section{Quality of Service (QoS)}

Quality of Service for the Voice over Internet Protocol is defined as a capacity for continuous, clear, and uninterrupted transmission and reception of voices [16]. Due to the four dominant limitations, QoS needs to be implemented:

- Guaranteed bandwidth support: Enough bandwidth is needed to support the traffic of voice and the data in real-time before the occurrence of voice.

- Jitter: It is the measurement of variable time to send and receive packets across a network for all network connections as the VOIP network.

- Latency: Delay and elapse most of the time is described through the length of time it possesses to reach the listener after being spoken or transmitted by the speaker [9].

\section{a. QoS in wired}

The primary method of queuing could not solve, neither the leading weighted fair queuing method could solve the problem of the quality of service caused by converged network traffic. If only a voice-enabled network priority is used so that voice is given a priority than data traffic. When WFQ is treated data fairly [10], voice still experiences a delay. All user classifications and queuing mechanisms are time-consuming and complicated to operate on every interface. New queue mechanisms have been developed for combining the best aspects of existing queuing processes.

\section{a) Class-Based Weighted Fair Queuing (CBWFQ)}

CBWFQ is a system for guaranteeing class bandwidth. WFQ standard expands through the support of class that is user-defined for the traffic. These kinds of classes are identical to the benchmark, including the commands of the Access Control List and the protocols. For each class, a queue is reserved. If class ID is described and matching its requirements, then we can give bandwidth and the maximum packet limit properties of the class. This bandwidth is observed during traffic congestion, and the number of classes is around 64 to deal with service policy.

\section{- Classification:}

Class maps are distributed through classification. The presence of concern classification choices is based on the version of IOS Cisco. It will depend on the interface format and the encapsulation which uses the service policies.

\section{- Architecture:}

CBWFQ supports the classification of traffic in its respective FIFO (First in First out) queues in various class maps. CBWFQ classes are treated with a tail drop if a weighted early random detection policy is used for the class to prevent congestion. The default queuing 
technique is overruled by empowering Class-Based Weighted Fair Queuing on a physical interface as shown in Fig.3.

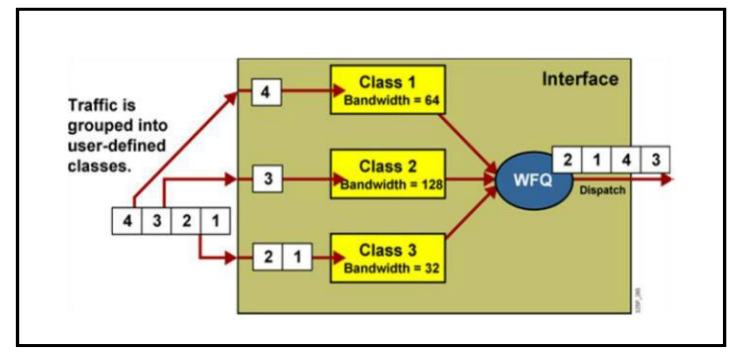

Fig.3. CBWFQ Architecture

- Scheduling:

CBWFQ provides a weight-allocated bandwidth for traffic classes. Percentage or the bandwidth is computed internally. It solely relies on the kilobits per second bandwidth, percentage of bandwidth and its remaining percentage [11].

Available bandwidth is calculated according to the following formula:

$$
\begin{gathered}
\text { Bandwidth }_{\text {avail }}=[\text { Bandwidth } * \text { MaxReservable- } \\
\text { SUM(all fixed guarantees })]
\end{gathered}
$$

\section{b. QoS in Wireless}

Wireless networks have become an essential factor in the latest year that is why Quality of Service participates in services that are wireless in order to offer the best quality of the video, data applications, and voice. Wireless networks can not achieve QoS techniques used in wired networks. There are many more limitation, among the few are:

1. Interference: Electric noise and frequency bands of the free variety used by wireless connection makes wireless signal suffer serious disturbance.

2. Hidden Node Problem: When it comes to wireless connectivity, where the access point is evident to the node, while it is not evident to some other nodes that communicate with AP.

3. Multipath Propagation: Due to diffraction, dispersion, and reflection, the signal might opt for the distinct routes among the receiver and the sender.

4. Handoff: It must reset the route possession which starts in wireless networks at the node of mobile [12].

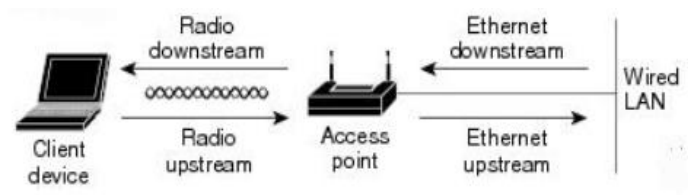

Fig.4. Downstream and Upstream Flow of Traffic

With the increment in load, Quality of Service has prioritized the traffic from Access Point via wireless network classification, mainly with regard to downstream prioritization compared with upstream traffic flows, which is provided in the fig.4 [13].

a) $802.11 e$

802.11e is a suggested improvement to the wireless LAN requirements of $802.11 \mathrm{a}$ and $802.11 \mathrm{~b}$. It provides QoS functionality including voice, video, and data transmission priority. The standards $802.11 \mathrm{e}$ belongs to the 802.11 families of wireless local area networks (WLAN) but It is estimated that $802.11 \mathrm{e}$ business [14] and consumer products are widely available in late 2004 or 2005. The LinkSys Access point has capability to add the suggested device by MAC and makes its voice, video or data priority with application of 802.11e.

\section{Methodology}

There are two cases were designed to analyses of voice, and data transmission by sustaining the quality as the voice cannot afford delay in any scenario wired or wireless. Bandwidth is assigned and increased according to needs but we had implemented QoS on both scenarios as discussed above CBWFQ for wired and 802.11e for wireless.

\section{CASE-1 Wired Scenario:}

As shown in Fig.5 we had developed two different LANs with IPs of (10.0.0.0 and 20.0.0.0) connected by a single Wide Area Networks (15.0.0.0), ATA worked for the conversion of Analog voice phone call to digital VoIP call, Computers performed netmeting as well as monitored the data rates of each VoIP call routed form LAN1(10.0.0.0) to LAN2(20.0.0.0) by PRTG then created congestion artificially by LAN Traffic Generator and applied QoS for wired as discussed above CBWFQ to sustain the quality as given dedicated bandwidth to voice with first high priority of network.

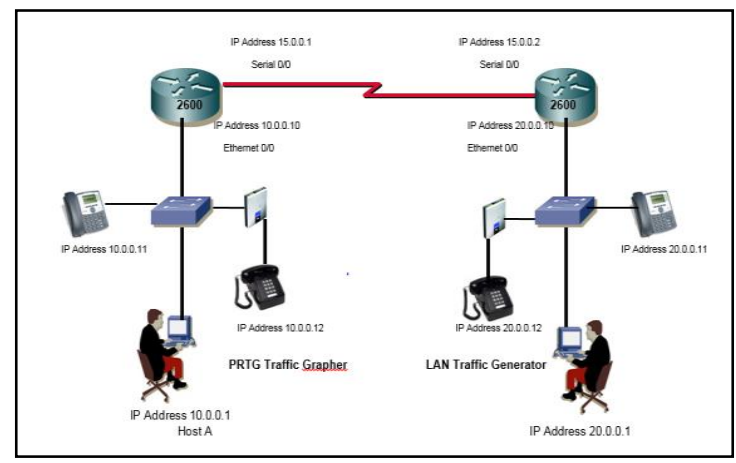

Fig.5. VoIP over Wired Network

\section{CASE-2 Wireless Scenario:}

As shown in Fig.6 we had created two different VLANs for wired network separated by two different IPs (10.0.0.0 and 20.0.0.0). Cisco Catalyst Switch was trunked by a single LinkSys Access Point to analyse the data rates of routed call from wired to wireless. With the same AP developed two different SSIDs named as (ESP- 
Marketing and ESP Finance) both have diferent IP addresses (10.0.0.0 and 20.0.0.0) similar to wired network collecteively two Local Networks were created with different client's properties (wired and wireless) to make the comparision easier from wired to wireless call and wireless to wireless call. PRTG and LAN Traffice Generator were used at both ends of clients.

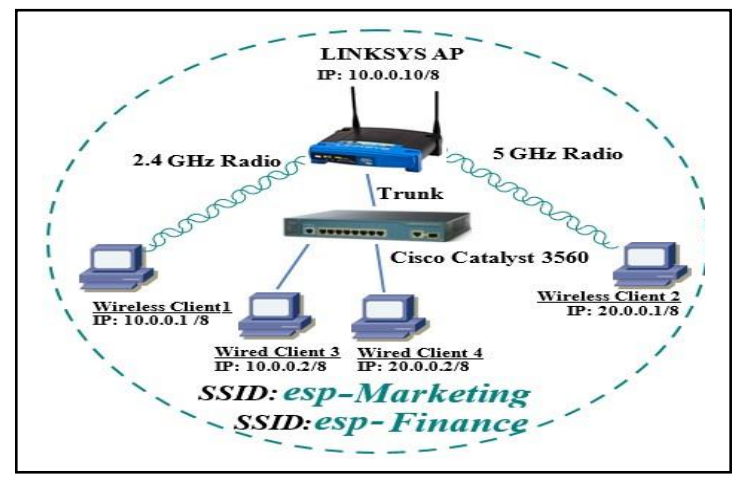

Fig.6. VoIP over a wireless Network

\section{RESUlTS AND DISCUSSION}

This section discusses the outcomes on PRTG and LAN Traffic Generator by implementing two scenarios wired and wireless as shown in figure in Fig.5 and Fig.6, the aim is to calculate the bandwidth of voice call, minimize it according to available bandwidth, and regulate the congestion by applying QoS techniques for VoIP.

\section{A. VoIP over wired network}

Primarily we analyzed the results on PRTG by performing (1-net-meeting +2 Voice over IP calls) on 125-kilo-bits per second bandwidth shown in the below Figure.7. The outcomes presents that all applications of voice hold 80-kilobits per second bandwidth and by using default codec scheme G.711u shown in Fig.8 in wired network while 30 to 40 kilobits per second bandwidth varies by net-meeting.

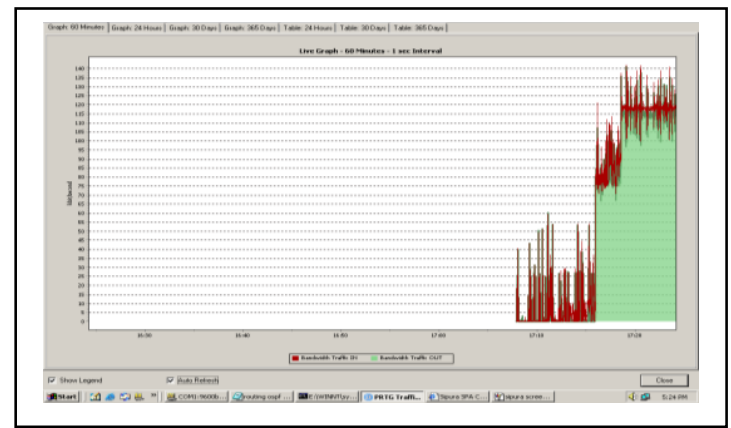

Fig.7. One-Net-meeting + Two VoIP calls on $125 \mathrm{Kbps}$ bandwidth

As shown in Fig.9 we changed the codec scheme from default G.711 to G.729 to minimize the bandwidth. It was monitored that VoIP $80 \mathrm{kbps}$ reduced to 20-kilobits per second for each VoIP call mentioned in Fig. 10.

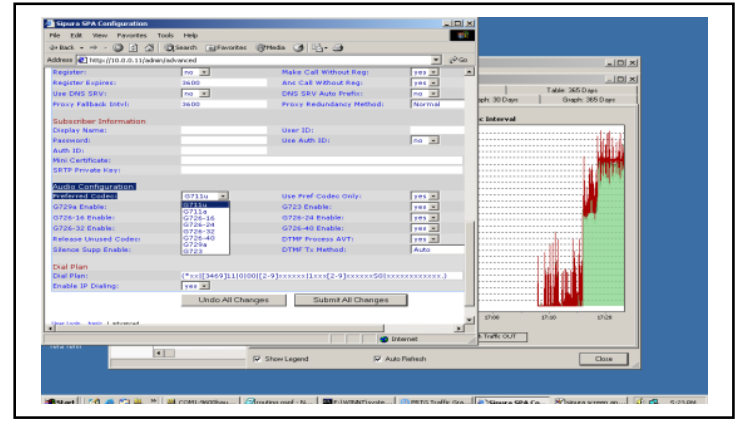

Fig.8. Default Codec scheme G.711u

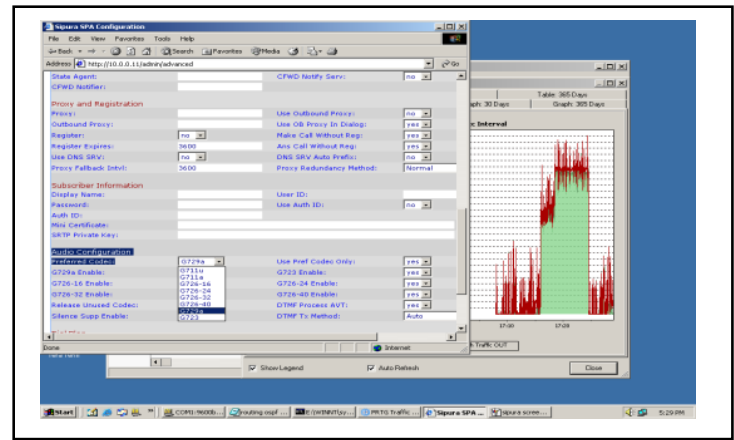

Fig.9. Change Codec scheme G.729

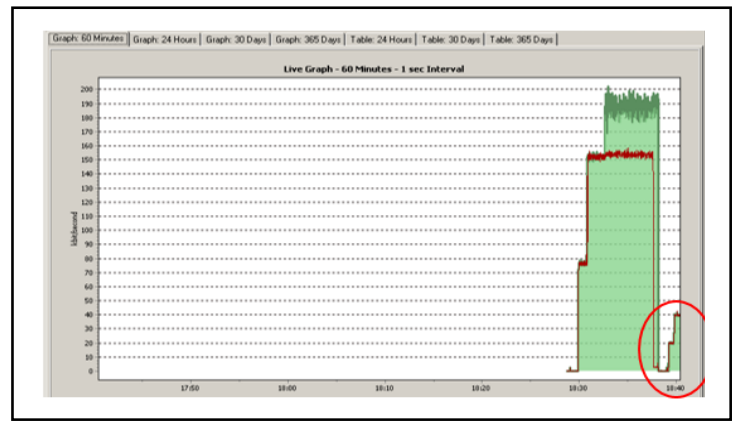

Fig.10. Two-VoIP calls with G.729 Codec

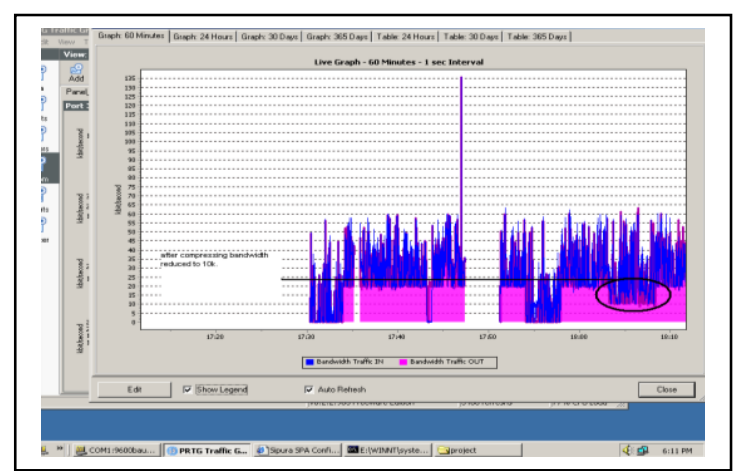

Fig.11. One-VoIP call with TRP Header Compression

\section{a. VoIP QoS (CBWFQ) over wired network}

In Fig. 12. Bandwidth of 192 kilobits per second was assigned to the DCE end of Cisco Router with that ClassBased Weighted Fair Queuing was done in the similar fashion as was mentioned above in Figure.5. Personal Computers were attached to the ends of the router to operate Local Area Network Traffic Generator. 80 kilobits per second bandwidth was dedicated to voice 
application in order to implement Class-Based Weighted Fair Queuing.

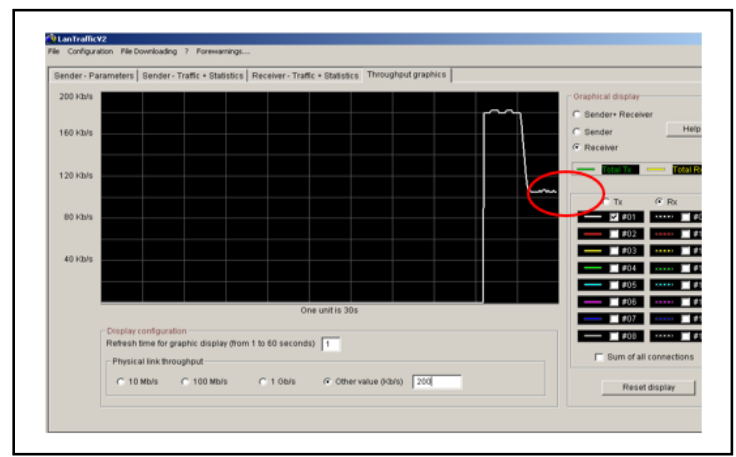

Fig.12. One-VoIP with QoS(CBWFQ)

In Fig.12 graph the bandwidth was occupied by another applications as dialing of Voice over IP call 80kilobits per second bandwidth went down on the first call according to the assigned bandwidth of voice. In a similar way, Fig.13 graph, two times bandwidth reduced by dialing 2 calls 80 -kilobits per second to assigned bandwidth for all VoIP calls.

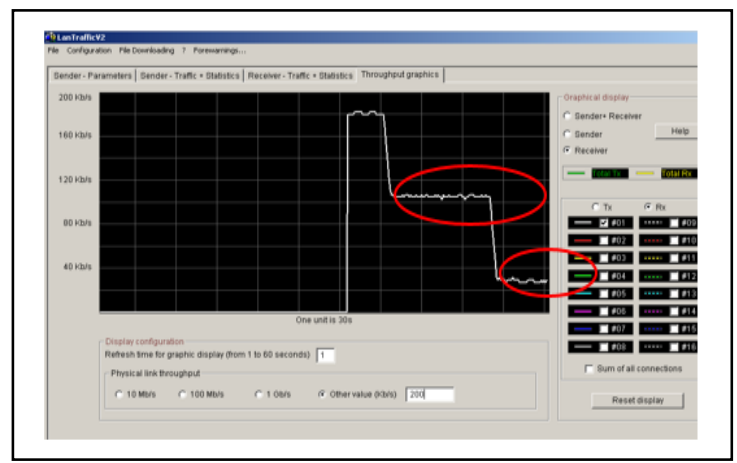

Fig.13. Two-VoIP with QoS(CBWFQ)

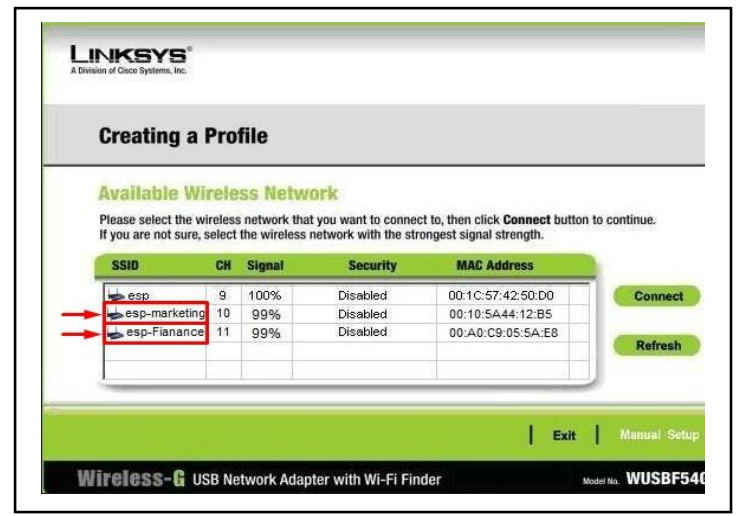

Fig.14. Two SSIDs with LinkSys Acess Point

\section{B. VoIP over Wireless}

In Fig.14 two SSIDs (ESP-Marketing and ESPFinance) were created on LinkSys Acess Point according to the wireless diagram given above in Fig.6. As routed the call wirelessly from ESP-marketing client to ESPfinance client the finding were almost same in Fig.15 as found in wired it proves that Voice over IP call always consume 70 to $80 \mathrm{Kbps}$ bandwidth over wired or wireless network.

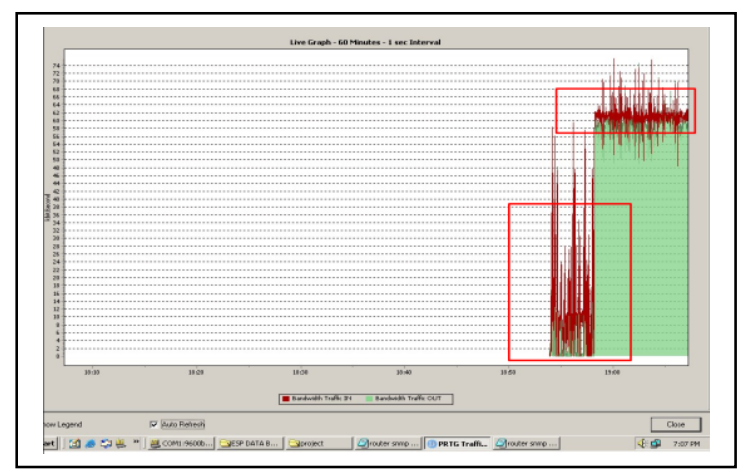

Fig.15. One-VoIP call + Net Meeting

\section{a. VoIP QoS (802.11e) over Wireless.}

As mentioned in Figure16. Quality of Service over the wireless network was achieved by Graphical User Interface of LINKSYS router, enabled IEEE standard 802.11e to prioritized the voice application high, device was generally named as ATA added the user MAC address can be seen in the Fig.16. It was examined that if congestion encountered in wireless this ATA device will always get high Voice priority than other traffic.

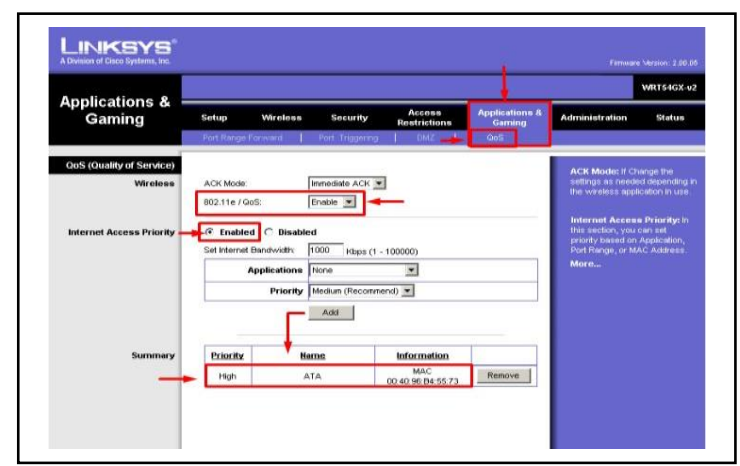

Fig.16. VoIP QoS on wireless

\section{CONCLUSION}

In this paper calculation, it was explored that VoIP quality is always good in wired owing to the absence of interference, delay and packet loss however the congestion was regulated by QoS (CBWFQ) in case of heavy network traffic. On the other side VoIP over wireless have some problem but it can readily controlled by certain QoS techniques such as given by $802.11 \mathrm{e}$ standard, in addition facilities were offered such as easy admittance, mobility, fast and cost-efficient. The consequences states that VoIP is possible over both wired and wireless networks but efficient use of bandwidth can reduce the cost. Clients are preferred to attempt VoIP over wired if the quality is essentials, it can take a longer time to implement and high cost than wireless. If mobility is essential such as in warehouse, universities and hospitals where wiring is typical to connect each user, 
need simple admittance, low cost and feasibility for smart devices, should offered Voice over Internet Protocol with wireless network that is the base of coming network technologies.

\section{FUTURE WORK}

In this paper analysis we worked on two QoS techniques CBWFQ for wired and 802.11e for wireless users. Furthermore this research can be extended to use newly established QoS methods such as LLQ (Low Latency Queuing) for wired and WMM (Wifi Multimedia) for wireless networks.

\section{ACKNOWLEGEMENT}

I would like to thanks to all mighty "Allah" who is most beneficent and most merciful.

\section{REFERENCES}

[1] C. Jackson, J. Shorter, and K. Forcht, "REPLACING TRADITIONAL TELEPHONY WITH VoIP," vol. 10, no. 2, 2009.

[2] H. M. Chong and H. S. Matthews, "Comparative analysis of traditional telephone and Voice-over-Internet Protocol (VoIP) systems," IEEE Int. Symp. Electron. Environ., pp. 106-111, 2004.

[3] S. Golhar and V. S. Dhamdhere, "Voice over Internet Protocol (VoIP) Based IP PBX System Design," Int. J. Sci. Res., vol. 5, no. 2, pp. 1380-1385, 2016.

[4] N. Chirdchoo, W. Cheunta, K. Saelim, and P. Kovintavewat, "Design and implementation of a VoIP system for campus usage: A case study at NPRU," 13th Int. Symp. Commun. Inf. Technol. Commun. Inf. Technol. New Life Style Beyond Cloud, Isc. 2013, pp. 216-221, 2013.

[5] M. Syed and M. N. S. Raza. Dilber, "Comparative analysis of traditional telephone and VoIP systems," $J$. Indep. Stud. Res. - Comput., vol. 12, no. 1, pp. 106-111, 2014.

[6] S. Jalendry and S. Verma, "A Detail Review on Voice over Internet Protocol (VoIP)," Int. J. Eng. Trends Technol., vol. 23, no. 4, pp. 161-166, 2015.

[7] H. Kazemitabar, S. Ahmed, K. Nisar, A. B. Said, and H. B. Hasbullah, "A survey on voice over IP over wireless LANs," World Acad. Sci. Eng. Technol., vol. 71, pp. 352358, 2010.

[8] A. H. M. Amin, "VoIP Performance Measurement Using QoS Parameters," Second Int. Conf. Innov. Inf. Technol., pp. 1-10, 2005.

[9] K. S. Mohamed, "VoIP Techniques and Challenges Karama Said Mohamed School of Engineering, Design and Technology."

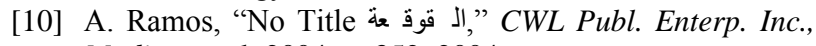
Madison, vol. 2004, p. 352, 2004.

[11] R. B. Bahaweres, A. Fauzi, and M. Alaydrus, "Comparative analysis of LLQ traffic scheduler to FIFO and CBWFQ on IP phone-based applications (VoIP) using Opnet (Riverbed)," Proceeding 2015 1st Int. Conf. Wirel. Telemat. ICWT 2015, no. 2, pp. 1-5, 2016.

[12] Gregarion Narvaez, "InfoSec Reading Room In tu, A ho $11 \mathrm{r}$ igh," Inf. Secur., 2007.
[13] N. Khademi and M. Othman, "Guaranteeing per station and per flow fairness of downstream and upstream flows over IEEE 802.11 WLAN," 2009 Int. Conf. Inf. Multimed. Technol. ICIMT 2009, no. 1, pp. 431-435, 2009.

[14] R. S. Sherratt and L. P. Gao, "Offering 802.11 MAC frame transport over Multiband OFDM for consumer devices," Proc. Int. Symp. Consum. Electron. ISCE, pp. 230-233, 2006.

\section{Authors' Profiles}

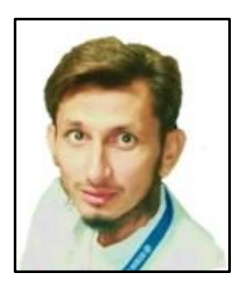

Zahid Ali- is RESEARCH ASSOCIATE in Department of Telecommunication Engineering at Mehran UET Jamshoro, Sindh, Pakistan as well as enrolled in Master Communication System \& Networks. $\mathrm{He}$ has completed his B.E of Telecommunication from Dawood UET, Karachi in 2016.

He has various experiences relevant to his field. From 20182019 worked at Basecamp Data Solution Multinational Company Ohio, USA as a position of BUSINESS PROCESS ASSOCIATE (IT-SUPPORT) to support cooperate clients (Verizon stores of I-Phone) located in USA. His research interest in Microwave, Modern Antennas, Communication Systems and Networks with the growth of Telecom Project Management \& Operation Maintenance.

Falak Naz- is a final year student from Department of Telecommunication. Mehran UET Jamshoro, Sindh, Pakistan. She has experienced to work on IoT projects, Microstrip Patch Antenna Design and currently working on Sierpinski Microstrip Patch Antenna for Smart Vehicles and now working on her final year project named as "Obstacle dictation and distance measurement using wifi”.

Her research interest is in Microwave, Antennas and Wireless Sensor Networks.

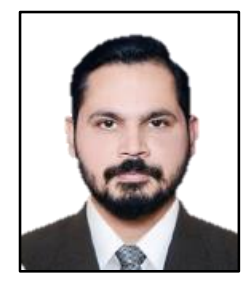

Javed- is currently a HUAWEI ICT AMBASSADOR and enrolled in Master Communication System \& Networks at Mehran UET Jamshoro, Sindh, Pakistan. He has completed his B.E of Computer System from QUEST Nawabshah in 2013.

He has different experiences relevant to Communication and Networking.

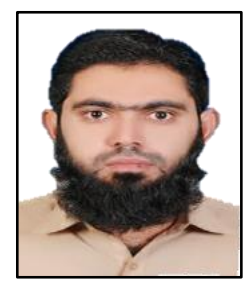

Muhammad Qurban- is currently a TEACHER in Educator Jamshoro Sindh, Pakistan as well as enrolled in Master Communication System \& Networks. He has completed his B.E of Telecommunication from Mehran UET in 2015.

He has experienced to work as TEAM LEAD on Telenor 3G ZTE project at Interaction International Group. His research interest in Computer, Communication and Networking. 


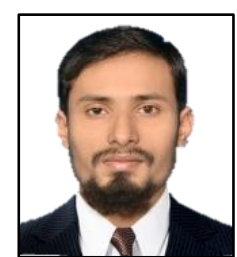

Muhammad Yasir- is currently enrolled in Master Communication System \& Networks at Mehran UET Jamshoro, Sindh Pakistan. He has completed his B.E of Telecommunication from Dawood UET Karachi in 2015.

ENGINEER at NETKC duration of 2016-2018. His research interest in Computer, Communication and Networking.

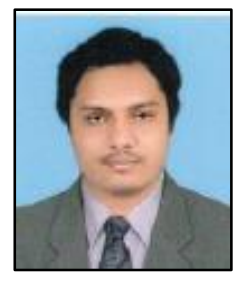

Shehroz Jehangir- is currently working as Research Assistant as well as enrolled in Telecommunication Engineering \& Management at Mehran UET Jamshoro, Sindh Pakistan. He has completed his B.E of Telecommunication from Mehran UET Jamshoro in 2015.

His research interest in Microwave, Antenna, Networking and Telecommunication.

How to cite this paper: Zahid Ali, Falak Naz, Javed, Muhammad Qurban, Muhammad Yasir, Shehroz Jehangir, "Analysis of VoIP over Wired \& Wireless Network with Implementation of QoS CBWFQ \& 802.11e", International Journal of Computer Network and Information Security(IJCNIS), Vol.12, No.1, pp.43-49, 2020. DOI: 10.5815/ijcnis.2020.01.05 